\title{
Increased activity of serotonin uptake in platelets in medication overuse headache following regular intake of analgesics and triptans
}

\author{
Ilya Ayzenberg • Mark Oberman · Kirsten Leineweber - Leonora Franke • \\ Min-Suk Yoon · Hans-Christoph Diener · Zaza Katsarava
}

Received: 1 December 2007 / Revised: 17 January 2008/Accepted: 18 January 2008/Published online: 11 March 2008

(c) Springer-Verlag 2008

\begin{abstract}
We investigated the effect of chronic administration of different pain medications on the activity of the serotonin transporter (SERT) in patients with medication overuse headache $(\mathrm{MOH})$. We measured the kinetic of platelet 5-HT uptake (maximal velocity, $V_{\max }$ and the Michaelis-Menten constant, $K_{\mathrm{m}}$ ) in patients with overuse of triptans (tMOH, $n=15$ ) or analgesics (aMOH, $n=14$ ) before and after drug withdrawal, as well as in headachefree healthy subjects $(n=15)$ and patients with episodic migraine (EM, $n=16)$. $V_{\max }$ was increased similarly in both, $\mathrm{tMOH}$ and aMOH compared to healthy subjects and patients with EM and normalized after withdrawal in parallel to the improvement of headache frequency. Average $K_{\mathrm{m}}$ was similar in all groups at baseline and not affected by the withdrawal. The data demonstrate a transient increase of SERT activity in patients with analgesic and triptan induced $\mathrm{MOH}$ but do not allow to differentiate whether the increase of serotonin uptake is caused by regular intake of analgesics or triptans or is a consequence of frequent headache attacks.
\end{abstract}

I. Ayzenberg $(\bowtie) \cdot$ M. Oberman · M.-S. Yoon · H.-C. Diener ·

Z. Katsarava

Department of Neurology, University of Duisburg-Essen, Hufelandstr. 55, Essen 45122, Germany

e-mail: ilya_ayzenberg@yahoo.com

K. Leineweber

Department of Pathophysiology, University of Duisburg-Essen, Hufelandstr. 55, Essen 45122, Germany

L. Franke

Department of Psychiatry and Psychotherapy, Charite University of Medicine, Berlin, Germany
Keywords Chronic migraine .

Medication overuse headache $\cdot \mathrm{MOH} \cdot$ Serotonin uptake . Platelets

\section{Introduction}

Transformation of episodic migraine to chronic daily or near-daily headache caused by an excessive use of medication taken for symptomatic headache relief is a well known phenomenon. Medication overuse headache $(\mathrm{MOH})$ has developed into the third most common type of headache after tension-type headache (TTH) and migraine [1]. All anti-headache drugs, such as triptans, analgesics, ergots, and opioids can induce $\mathrm{MOH}$.

Clinically, triptan induced chronic migraine differs from chronic migraine following the overuse of analgesics. Overuse of triptans can cause migraine-like daily headaches (as opposed to analgesics that usually cause tension type like daily headache) but also might solely cause a considerable increase in migraine attack frequency without necessarily causing daily headache. Additionally, the overuse of triptans leads to $\mathrm{MOH}$ faster and with lower dosages compared to ergots or analgesics, while it is also characterized by shorter and easier withdrawal with lower relapse rates compared to analgesics overuse [2].

Despite a growing scientific interest, the pathophysiological mechanisms associated with the development of $\mathrm{MOH}$ are still insufficiently understood. One of the key pathophysiological mechanisms could involve the alteration of the serotonin metabolism. Abnormalities of 5-HT turnover have been demonstrated in patients with chronic headache due to analgesic overuse [3-6]. It is not known, however, whether the overuse of triptans leads to the alteration of the serotonin metabolism as well. 
In the present study we aimed to investigate the serotonin metabolism in patients with triptan- and analgesics induced $\mathrm{MOH}$. We therefore studied the functional properties of the platelet serotonin transporter (SERT) in patients with triptan $\mathrm{MOH}(\mathrm{tMOH})$ and analgesic $\mathrm{MOH}$ $(\mathrm{aMOH})$ and compared it to patients with episodic migraine and headache-free healthy subjects. We used the platelet model for several reasons: SERT in all tissues and organs, including brain, gut and platelets are encoded by the same gene and to date, there is no definitive evidence for the existence of a distinct tissue-specific SERT [7]. Therefore, platelets have been widely used as a practical, easily accessible and reproducible peripheral model for the study of SERT functions and its alterations. A recent study indicated that there is a relationship between the SERT measures in platelets and in the human midbrain [8].

Given the prior reports, we hypothesized that overuse of triptans and analgesics may cause alterations in SERT function, which would be reversible after drug withdrawal. We moreover aimed to find differences between patients overusing analgesics and patients overusing triptans.

\section{Methods}

The study protocol was approved by the ethics committee of the University of Duisburg-Essen. Subjects were recruited from our headache clinic; healthy controls consisted of University of Duisburg-Essen medical students and faculty members. All participants gave their written informed consent according to the Declaration of Helsinki. All participants received a standard interview and neurological examination. Inclusion criteria were: age between 18 and 65 years and primary migraine type of headache according to the IHS criteria [9]. Exclusion criteria were suspicion for symptomatic headache, primary tension-type headache, co-morbid depression ( $>18$ according to Beck depression scale), overuse of two or more different types of acute headache drugs simultaneously and previous or actual treatment with antidepressants. Patients with chronic migraine with probable medication overuse headache who did not improve 6-8 weeks after withdrawal therapy were excluded as well.

The study population was divided into four groups:

Group 1 -healthy controls $(n=15)$

Group 2-episodic migraine without aura $(n=16)$

Group 3-analgesics induced medication overuse headache $(\mathrm{aMOH})(n=14)$

Group 4-triptan induced medication overuse headache $(\mathrm{tMOH})(n=15)$.

Patients with analgesics (group 3) and triptan (group 4) $\mathrm{MOH}$ were studied before and 6-8 weeks after withdrawal.
All patients (groups 2, 3 and 4) were studied outside the migraine attack. The time interval between the last migraine attack and blood sampling was at least 3 days. Venous blood was collected from the cubital vein into EDTA (1.6 mg EDTA/ml blood) vacutainer tubes between 8:00 and 9:00 a.m. after overnight fasting. After centrifugation at $250 \mathrm{~g}$ and $20^{\circ} \mathrm{C}$ for 15 min the supernatant of the platelet-rich plasma (PRP) was collected and subsequently the number of platelets in the PRP was counted. 5-HT uptake was studied within $1 \mathrm{~h}$ after the sample collection. Further sample processing was performed according to Thies-Flechtner et al. [10]. Briefly, aliquots of $50 \mu \mathrm{l}$ PRP were incubated in Krebs phosphate buffer at six different concentrations of $\left[{ }^{3} \mathrm{H}\right]-5-\mathrm{HT} / 5-\mathrm{HT}$ ranging from 0.1 to $1.6 \mu \mathrm{M}$, at $37^{\circ} \mathrm{C}$ and $4^{\circ} \mathrm{C}$ for $5 \mathrm{~min}$. The uptake was terminated by dilution of the samples with ice cold buffer and rapid filtration through Whatmann GF/B filters. Kinetic constants for uptake measurement (maximal velocity $\left(V_{\max }\right)$ and Michaelis-Menten constant $\left.\left(K_{\mathrm{m}}\right)\right)$ were obtained by direct weighted nonlinear regression of uptake rate against $\left[{ }^{3} \mathrm{H}\right]-5-\mathrm{HT} / 5-\mathrm{HT}$ concentrations with GraphPad PRISM software (GraphPad, San Diego, USA).

The variables were tested for normal distribution using the Kolmogoroff-Smirnoff test. One factorial ANOVA was used to compare mean values of $V_{\max }$ and $K_{\mathrm{m}}$ between groups. $\quad$ Factor $=$ GROUP: healthy controls versus episodic migraine versus analgesics induced $\mathrm{MOH}$ $(\mathrm{aMOH})$ versus triptan induced $\mathrm{MOH}$ (tMOH). Post-hoc comparisons were performed using the least statistical significances. A paired $t$-test was used to compare the results of patients with analgesics and triptans induced $\mathrm{MOH}$ before and after withdrawal. Spearman correlation coefficients were calculated for testing for possible relationships between headache frequency and kinetic characteristics of 5HT uptake in platelets. All statistics were calculated with SPSS 13.0 (SPSS Inc. Chicago, IL, USA). The level of significance was set at $p<0.05$.

\section{Results}

General group characteristics

Demographic and clinical characteristics of the study population are given in Table 1. There were no significant differences between the groups considering age and gender. Among the MOH patients, the monthly headache days ranged from 18 to 30 days. In average, the frequency of headache was not different between $\mathrm{tMOH}$ and $\mathrm{aMOH}$ patients (Table 1). Eleven of 14 patients with aMOH and 13 of 15 patients with tMOH improved significantly (reduction of the headache frequency by more than 50\%) after drug withdrawal. 
Table 1 Demography and clinical characteristics of the study population

\begin{tabular}{llllclcccc}
\hline Groups & $N$ & Age & Women & BDI & $\begin{array}{l}\text { Duration of } \\
\text { migraine } \\
\text { (years) }\end{array}$ & $\begin{array}{l}\text { Duration of } \\
\text { MOH } \\
\text { (years) }\end{array}$ & $\begin{array}{l}\text { Headache } \\
\text { frequency } \\
\text { (days/month) }\end{array}$ & $\begin{array}{l}\text { Medication intake } \\
\text { frequency } \\
\text { (days/month) }\end{array}$ \\
\hline Controls & 15 & $39.6 \pm 12.4$ & 11 & - & - & - & - & - \\
Episodic migraine & 16 & $37.4 \pm 12.2$ & 13 & $5.1 \pm 2.7$ & $21.4 \pm 10.4$ & - & $4.37 \pm 1.82$ & - \\
Analgesic induced MOH & 14 & $39.3 \pm 16.5$ & 11 & $6.9 \pm 3.9$ & $23.3 \pm 9.6$ & $3.99 \pm 3.34$ & $23.7 \pm 4.7$ & $23.5 \pm 4.6$ \\
Triptan induced MOH & 15 & $40.9 \pm 11.8$ & 13 & $6.3 \pm 4.4$ & $24.9 \pm 10.3$ & $3.44 \pm 3.1$ & $22.1 \pm 6.0$ & $19.1 \pm 7.6$ \\
\hline
\end{tabular}

$\mathrm{MOH}$ medication overuse headache, $B D I$ Beck-Depression-Inventory

\section{HT uptake in platelets}

There were no significant differences in mean $V_{\max }$ values between patients with episodic migraine and healthy controls $\left(168.3 \pm 64.8\right.$ vs. $190.3 \pm 70.9 \mathrm{pMol} / 10^{8}$ plat $\times$ min). In contrast, mean $V_{\max }$ was significantly higher in $\mathrm{aMOH}$ and $\mathrm{tMOH}$ groups, being $274.9 \pm 97.8$ and $285.2 \pm 90.6 \mathrm{pMol} / 10^{8}$ plat $\times$ min respectively $(d f=3$, $F=8.19, p<0.05$ ) (Fig. 1).

After withdrawal $V_{\max }$ normalized in both groups and reached the level of healthy controls: $210.7 \pm 56.5$ in $\mathrm{aMOH}$ and $200.2 \pm 92.5$ in $\mathrm{tMOH}$ patients. The Michaelis-Menten constant $K_{\mathrm{m}}$ was found to be very similar in all four groups and not influenced by drug withdrawal in $\mathrm{MOH}$ patients (Fig. 2). No correlation was found between the headache frequency and 5HT uptake rate in both tMOH and aMOH groups separately as well as in overall $\mathrm{MOH}$ patients.

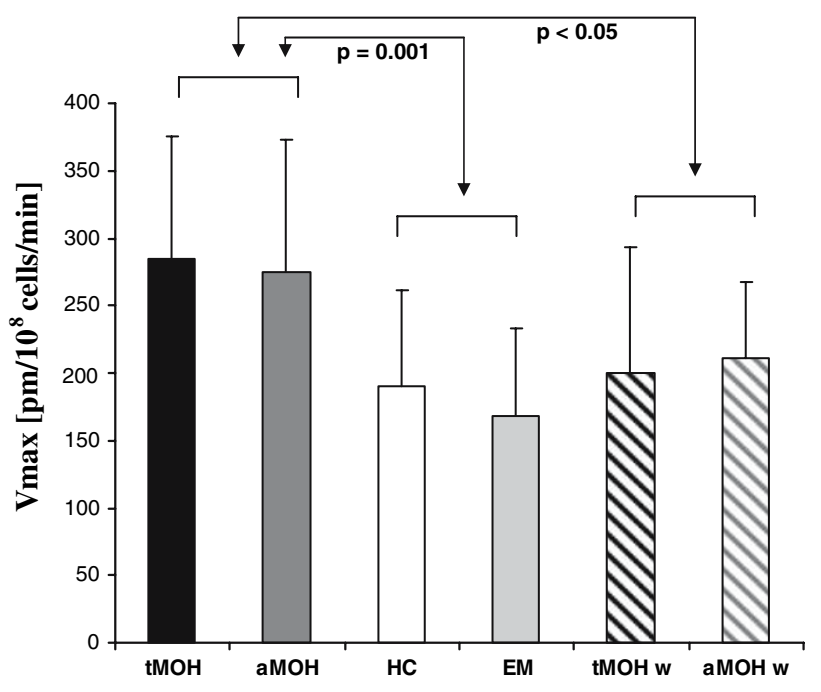

Fig. 1 Kinetic activity of the serotonin (5-HT) transporter for all investigated groups $\left(V_{\max }=\right.$ maximal velocity). Triptan induced medication overuse (tMOH), analgesics induced medication overuse headache $(\mathrm{aMOH})$, healthy controls $(\mathrm{HC})$, episodic migraine $(\mathrm{EM})$, tMOH after withdrawal therapy (tMOH w) and aMOH after withdrawal therapy (aMOH w)

\section{Discussion}

We investigated the serotonin turnover in platelets in patients with $\mathrm{MOH}$ following overuse of triptans and analgesics before and after withdrawal. The main findings of our study are: (a) patients with both, analgesic or triptan induced medication overuse headache had higher 5-HT uptake rates than patients with episodic migraine and controls; (b) this normalized again after withdrawal in both groups; (c) there were no differences between patients with analgesic induced $\mathrm{MOH}$ and triptan induced $\mathrm{MOH}$.

Acute administration of both, acetaminophen and acetylsalycilic acid caused significant increase of brain serotonin (5-HT) content and decrease of the number of 5-HT2 receptors in cortical brain membranes [11, 12]. Serotonergic mechanisms are thought to play an important role as brain serotonin depletion prevented the antinociceptive effect of acetaminophen. The chronic administration of acetaminophen however, resulted in a significant decrease in the maximum number of 5-HT2A binding sites and an increase in the maximum number of 5HT transporter binding sites in frontal cortical membrane [13], which is line with our results. Reduced serotonin levels and diminished endogenous cannabinoid levels in platelets of chronic migraine and $\mathrm{MOH}$ patients were

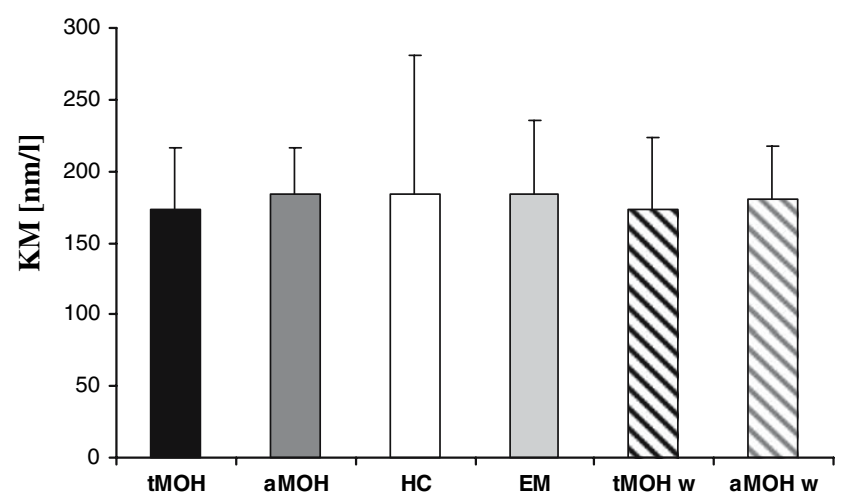

Fig. 2 Michaelis-Menten-Constant $\left(K_{\mathrm{m}}\right)$ reflecting binding affinity of the 5-HT transporter. No changes were seen across groups 
shown by other groups only recently and provide further evidence for a dysfunctioning of these systems in chronic headache [6].

Clinical efficacy of triptans is related to their vascular, neuronal and central effects at the $5 \mathrm{HT}_{1 \mathrm{~B} / 1 \mathrm{D} / 1 \mathrm{~F}}$ receptors [3]. As observed in different experimental conditions, triptan-sensitive $5 \mathrm{HT}_{1 \mathrm{~B} / 1 \mathrm{D}}$ receptors are predominantly terminal autoreceptors inhibiting 5HT release and/or synthesis and decreasing brain 5HT level [14, 15]. Acute, systemic application of sumatriptan and zolmitriptan in rats was found to decrease the 5HT synthesis in many brain regions, including the dorsal raphe nucleus. Chronically applied, the drugs induced a significant increase of 5HT synthesis in many projection areas but not in the dorsal raphe nucleus [14]. Reuter et al. [16] demonstrated that chronic sumatriptan and zolmitriptan treatment (2 weeks) significantly reduces $5 \mathrm{HT}_{1 \mathrm{~B} / 1 \mathrm{D} / 1 \mathrm{~F}}$ mRNA in trigeminal ganglion cells. Thus, excessive use of anti-migraine medication may result in alterations of serotonergic neurotransmission. However, to our knowledge there are no studies investigating the effect of chronic triptan administration on serotonin uptake.

Alternatively, changes of serotonin metabolism may reflect the consequence of pain chronification in headache patients. Serotonin release during the migraine attack is a well known phenomen and platelets serotonin depletion was demonstrated in chronic headache due to analgesics overuse previously. After withdrawal the headache frequency decreased and platelet serotonin normalized $[4,5$, 17]. Similarly in our study we demonstrated up-regulation of serotonin uptake in $\mathrm{MOH}$ patients before withdrawal and its normalization after withdrawal with no differences between the analgesic and triptan group. This observation is in line with a recent, in vivo ${ }^{123}$ I-ADAM SPECT study that demonstrated significantly higher SERT binding in the brainstem of migraine patients during attack-free period compared to healthy subjects: the monthly migraine days, ranged from 2 to 17 days, and the ADAM binding ratio in the brainstem correlated significantly [18].

We therefore conclude that: (a) SERT activity is increased in patients with medication overuse headache; (b) it is reversible and normalizes after withdrawal; (c) based on our data we are not able to differentiate whether the increase of serotonin uptake is caused by regular use of analgesics or triptans or is a consequence of frequent headache attacks. Further studies are needed to identify the precise mechanism responsible for the demonstrated SERT up-regulation in $\mathrm{MOH}$.

\section{References}

1. Celentano DD, Stewart WF, Lipton RB et al (1992) Medication use and disability among migraineurs: a national probability sample. Headache 32:223-228

2. Limmroth V, Katsarava Z, Fritsche G, Przywara S, Diener HC (2002) Features of medication overuse headache following overuse of different acute headache drugs. Neurology 59:10111014

3. Goadsby PJ (2000) The pharmacology of headache. Prog Neurobiol 62:502-525

4. Hering R, Glover V, Pattichis k, Catarci T, Steiner TJ (1993) 5HT in migraine patients with medication-induced headache. Cephalalgia 13:410-412

5. Srikiatkhachorn A, Maneesri S, Govitrapong P, Kasantikul V (1998) Derangement of serotonin system in migraineous patients with analgesic abuse headache: clues from platelets. Headache 38:43-49

6. Rossi C, Pini LA, Cupini ML, Calabresi P, Sarchielli P (2007) Endocannabinoids in platelets of chronic migraine patients and medication-overuse headache patients: relation with serotonin levels. Eur J Clin Pharmacol Nov 15; [Epub ahead of print]

7. Malmgren R, Hasselmark L (1988) The platelet and the neuron: two cells in focus in migraine. Cephalalgia 8:7-24

8. Uebelhack R, Franke L, Herold N, Plotkin M, Amthauer H, Felix $\mathrm{R}$ (2006) Brain and platelet serotonin transporter in humanscorrelation between [123I]-ADAM SPECT and serotonergic measurements in platelets. Neurosci Lett 406(3):153-158

9. The international classification of headache disorder (2004) 2nd Edn. Cephalalgia 24(Suppl 1):9-160

10. Thies-Flechtner K, Weigel I, Muller-Oerlinghausen B (1994) 5-HT uptake in platelets of lithium-treated patients with affective disorders and of healthy controls. Pharmacopsychiatry 27(Suppl 1):4-6

11. Pini LA, Sandrini M, Vitale G (1996) The antinociceptive action of paracetamol is associated with changes in the serotonergic system in the rat brain. Eur J Pharmacol 308(1):31-40

12. Pini LA, Vitale G, Sandrini M (1997) Serotonin and opiate involvement in the antinociceptive effect of acetylsalicylic acid. Pharmacology 54(2):84-91

13. Srikiatkhachorn A, Tarasub N, Govitrapong P (2000) Effect of chronic analgesic exposure on the central serotonin system: a possible mechanism of analgesic abuse headache. Headache 40:343-350

14. Dobson CF, Tohyama Y, Diksic M, Hamel E (2004) Effects of acute or chronic administration of anti-migraine drugs sumatriptan and zolmitriptan on serotonin synthesis in the rat brain. Cephalalgia 24:2-11

15. Mitsikostas DD, Papadopoulou-Daifotis Z, Sfikakis A, Varonos D (1996) The effect of sumatriptan on brain monoamines in rats. Headache 36(1):29-31

16. Reuter U, Salomone S, Ickstein GW, Waeber C (2004) Effects of chronic sumatriptan and zolmitriptan treatment on 5-HAT receptor expression and function in rats. Cephalalgia 24:398-407

17. Srikiatkhachorn A, Anthony M (1996) Platelet serotonin in patients with analgesic-induced headache. Cephalalgia 16:423-426

18. Schuh-Hofer S, Richter M, Geworski L, Villringer A, Israel H, Wenzel R, Munz DL, Arnold G (2007) Increased serotonin transporter availability in the brainstem of migraineurs. J Neurol 254(6):789-796 